\title{
Wireless Remote Controlled POV Display
}

\author{
Akshay Dhruv \\ Student, Dept of EXTC \\ D. J. Sanghvi College of \\ Engineering \\ Mumbai
}

\author{
Dishant Shah \\ Student, Dept of EXTC \\ D. J. Sanghvi College of \\ Engineering \\ Mumbai
}

\author{
Darshil Shah \\ Student, Dept of EXTC \\ D. J. Sanghvi College of \\ Engineering \\ Mumbai
}

\author{
Amey Raikar \\ Student, Dept of EXTC \\ D. J. Sanghvi College of Engineering \\ Mumbai
}

\author{
Shivani Bhattacharjee \\ Professor, Dept of EXTC \\ D. J. Sanghvi College of Engineering \\ Mumbai
}

\begin{abstract}
The purpose of this project is to design and implement a remote controlled persistence-of-vision (POV) display that will be able to display multiple patterns based on remote input. Persistence of vision (POV) refers to the phenomenon in which the human eye persist the image for 1/16th of a second even after the removal of image. A POV display exploits this phenomena by spinning a one dimensional row of LED's through a two dimensional space at such a high frequency that a two dimensional display is visible. The speed at which the LED's rotate is fast enough such that the human eye perceives a two dimensional image.
\end{abstract}

\section{Keywords}

Microcontroller, Motor, Rotor, POV Display, Led Array, Infrared Transmitter \& Receiver.

\section{INTRODUCTION}

The basic theory behind POV is that if LEDs flash at the exact same location on each revolution and the rotating speed of the motor is fast enough, human eye is unable to detect the LEDs' downtime between flashes, and has the illusion that they are constantly on. A persistence of vision (POV) refers to the phenomenon of the human eye in which an afterimage exists for a brief time $(10 \mathrm{~ms})$.

Persistence of vision plays a vital role in keeping the world from going pitch black every time we blink the eyes. Whenever a ray of light strikes the retina of the eye, the brain holds the impression of that light for about a 10th to a 15th of a second after the source of that light is removed from sight. As a result, the eye cannot clearly distinguish changes in light that occur faster than this retention period. The changes go unnoticed or they appear to be one continuous picture to the human observer. This fundamental fact of the way human eye see is used to build the project [1]. The POV display will allow users to upload an image to be displayed through wireless communication. In this case, we created a cylindrical display by spinning a column of LED's around a motor shaft placed at the centre. The rotational speed of the LED's is fast enough such that the human eye perceives a two dimensional image. Since the idea is to make a portable POV display - weight, size and balance play crucial roles in this design. The plan is to make the display as a commercial ready product.

The primary components of the display are a motor and a rotor. The rotor is mainly consisted of a microcontroller and a row of LEDs. The microcontroller controls LEDs. The overall design of this project can be grouped in the following categories: hardware design which includes electrical and mechanical design, and software design [2], [9]. The mechanical design portion of this project required most intensive labor while the software and electrical design appear trivial. The hardware integration with an adept firmware proved to be the biggest challenge of all. Another challenge faced was mounting the electrical components onto the mechanical structure - i.e. the spinning arm. The mechanical design introduced in this project had various safety issues that also had been taken into consideration.

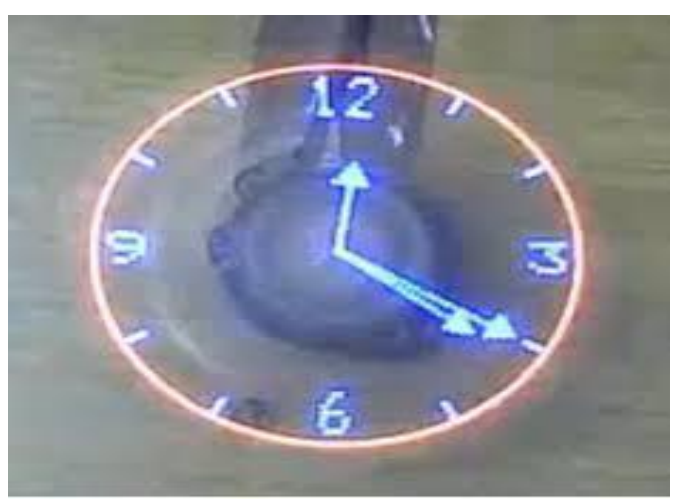

Fig1: Analog Clock

\section{NEED OF THE PROJECT}

The biggest advantage of this project is in the rotating display board which is a common seen nowadays. Advertisement is going digital. The use of led rotating display board at big shops, shopping centre's, bus stands, educational institutes and railway stations is becoming an effective mode of communication in providing information to the people.

The more attractive the message will be the more people will get profoundly influenced by it. The main purpose of this project is to make the advertising media an effective one. Following are the areas in which POV display plays a unique role:

\section{Advertising}

2. Education

3. Entertainment

4. Animation

5. Propeller Clock

6. Toys and Games

7. Malls

8. 3-D Display 


\section{THEORETICAL BACKGROUND}

In movies, a motion picture creates an illusion of a constantly lit screen by flashing individual photographs fast enough in succession. Even though the movie screen appears to be constantly lit, it is actually the dark segment of the time. It was this flickering image on the screen that gave rise to the term flicks in the early days of motion pictures. For a flicker-free picture today's motion pictures flash images on the screen at 24 frames per second (or 48 , in that each frame is flashed twice) Television, too, uses a complicated form of intermittent light impulses to literally build the picture. If an image can be form fast enough, the eye will be unaware about the occurrence of this process. In fact television in America can transmits and recreates 30 complete images per second to give the illusion of a single continuous picture [3], [7].

\section{METHODOLOGY, TECHNIQUES AND DESIGNING}

\subsection{Working Principle}

Rotation of single LED with high speed in circular motion gives the appearance of complete circle due to the persistence of vision (POV). A matrix id formed and the resolution of message depends on the rotational speed. The single element shows a pixel of the matrix. This idea can be further extended to create a display of letters or numbers by rotating a line of number of LEDs. Now on the basis of what has to be displayed, the LEDs are synchronized and the required message is displayed in two dimensions [1], [4].

The stand of the system will be a box inside which the DC motor is placed. The motor has its shaft sticking out of the box to the middle of box surface. Two layers of coils sit around this shaft. The inner coil closely wraps around a plastic cylindrical structure that can accommodate the motor shaft. This structure is also glued to the bottom of the rotor. When motor spins, it drives the cylindrical structure to spin along, hence the rotor. The rotor has two different circuit boards that are put together with two wood beams glued to them for support. One circuit board is the microcontroller PCB, and the other is an electrical board where LEDs and other circuits are soldered. The rotor and the motor can be separated [2].

\subsection{Hardware Design}

There are mainly two parts of the hardware design, motor circuit and rotor circuit. Motor circuit is powered by $5 \mathrm{~V}$ generator while the rotor circuit is powered by a small transformer which has an outer coil and an inner coil. The rotor sits on the motor's shaft and the shaft is surrounded by inner coil and outer coil. With the help of transformer, a battery is no longer needed to power rotor circuit, thus cutting down the weight of rotor. Motor circuit sets up the Infrared LED for the Infrared (IR) receiver to detect cycle time, time required for one rotation. It also converts the DC from a DC generator to AC. The AC flows in the outer coil and induces an $\mathrm{AC}$ in the inner coil. The rotor takes the AC and does a conversion to DC to power the microcontroller and the LEDs. The rotor also has two IR receivers, one of which is used for detecting a complete cycle and the other one is responsible for receiving remote control [2].

\subsection{Software Design}

The software allows generating different display patterns: a static heart image, an analog clock, a modifiable digital clock and a rotatable phrase "POV DISPLAY".
Our POV has a 2-degree resolution. This is achieved by splitting cycle time into 180 time intervals. Every $1 / 180$ of this time, LEDs are switched. The software is heavily consisted of math equations, interrupt service routines (ISR) and function calls. To enable all the functionalities for this project, four timers and two external interrupts are used [2]. There are two files used for the software design. One will be Main file and the other will be IRReceive file. IRReceive file detects key press from the remote control. Main file displays according to corresponding key press. There are four timers and two external interrupts used for this project. Two of the timers, timer1 and timer3 are responsible for the 2-degree resolution. Timer0 generates a lus time base. Timer 2 is used for IR communication between remote controller and IR receiver on the rotor.

\subsection{Block Diagram}

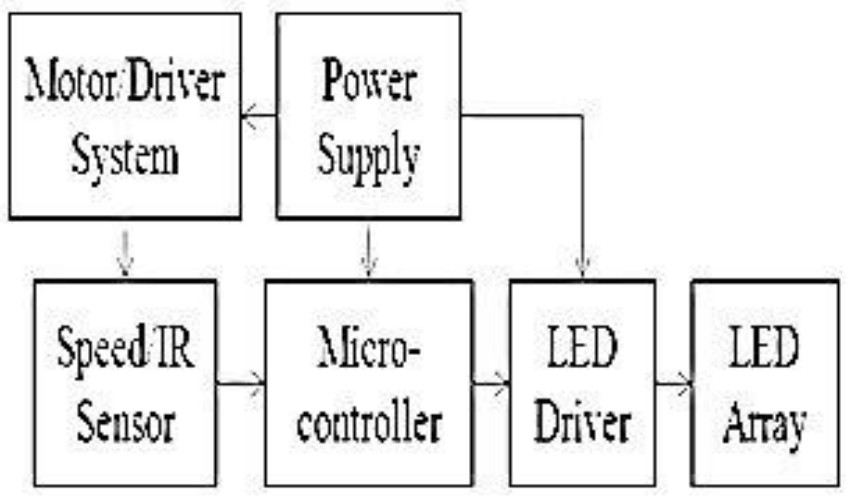

Fig2: Block Diagram of POV Display

\subsection{Description of Project}

Microcontroller: The microcontroller will act as an SPI master. It will communicate to the LED driver via SPI bus. It will send serial commands to write the registers on the LED driver chips.

LED driver: The LED-driver controls the functioning of LED array and provides the desired switching of LEDs.

LED Array: It is an array of LED that will turn on/off according to the message that is to be displayed. It displays the message in 2-D or 3-D pattern.

Motor/Driver System: This will provide the facility to rotate the onboard circuit up to the desired speed that is sufficient for the principle of Persistence of Vision.

Power Supply: The power supply unit provides the power to microcontroller, led driver and motor/driver system.

Sensor circuit and controller: To collect the RPM data needed for timing calculations and to change the display, the microcontroller is connected to a sensor circuit board. This circuit will sense the rotation and will provide the information about the revolution per second as well as the message displayed [3], [8].

\subsection{Schematics}

\subsubsection{Motor Circuit}

The motor circuit has four parts: 5V DC power supply, motor, infrared transmitter and LC circuit. The motor is connected to the power supply through a diode, preventing the reverse current generated from the motor when shut down from damaging other electrical components in the circuit. The infrared LED combined with the receiver on the rotor 
side is used to detect the cycle time of rotor. It has a $470 \mathrm{ohm}$ resistor connected to it for protection. The generator is connected to a 470uf capacitor for stabilization.

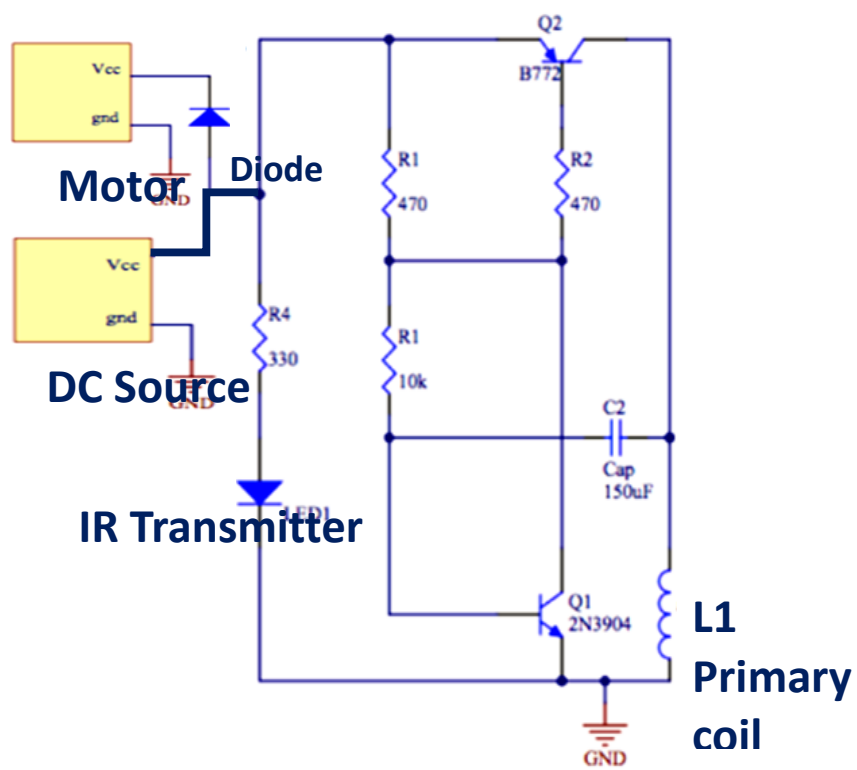

Fig 3: Motor Circuit

\subsubsection{Rotor Circuit}

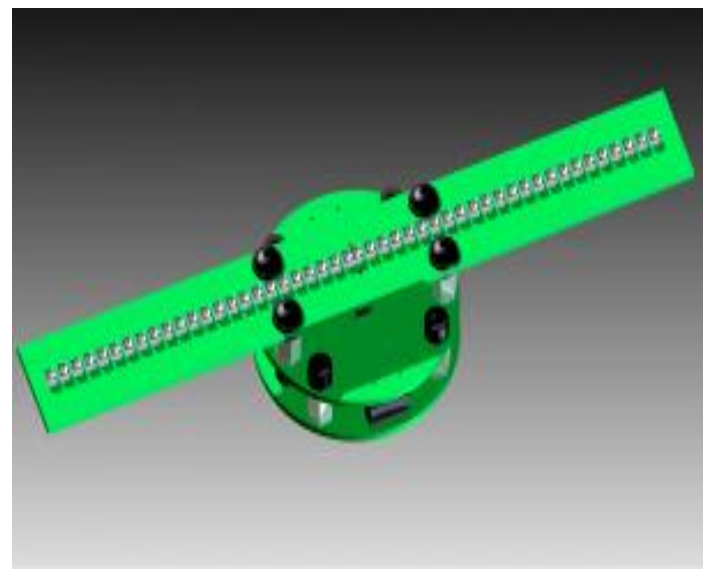

Fig 4: Rotor Circuit

The rotor circuit has many parts connected together, the microcontroller, LED array, AC to DC convertor, and Infrared receiver.

\subsubsection{Transformer Circuit}

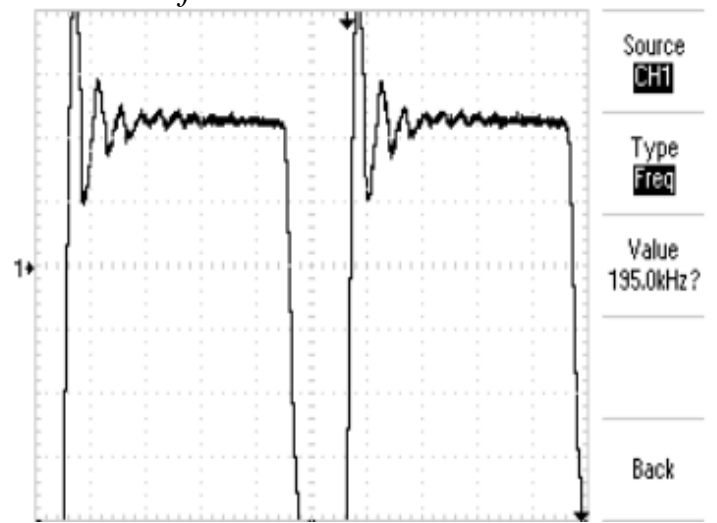

Fig5: Voltage of Primary Coil after DC to AC Conversion
The most important part in the motor circuitry is the LC circuit that converts DC to AC. A LC circuit can store energy oscillating at its resonant frequency $195 \mathrm{kHz}$. The power transistors used in the circuit are there to amplify signals and compensate power loss in the circuit. While running, the motor circuit draws $0.35 \mathrm{~A}$ current while the rotor circuit draws $0.1 \mathrm{~A}$ current. Hence, the power efficiency of such transformer is calculated to be $(5 \mathrm{~V} * 0.1 \mathrm{~A}) /(5 \mathrm{~V} * 0.35 \mathrm{~A})=$ $28.6 \%$. As the LEDs on the rotor require little current to function, such efficiency is sufficient to power all our LEDs and other electrical components. The outer and inner coil is careful chosen insulating copper wires and each wounded to about 60 rounds. The diameters for both coils are about $2 \mathrm{~cm}$. Multiple attempts were made here until the windings can provide enough power.

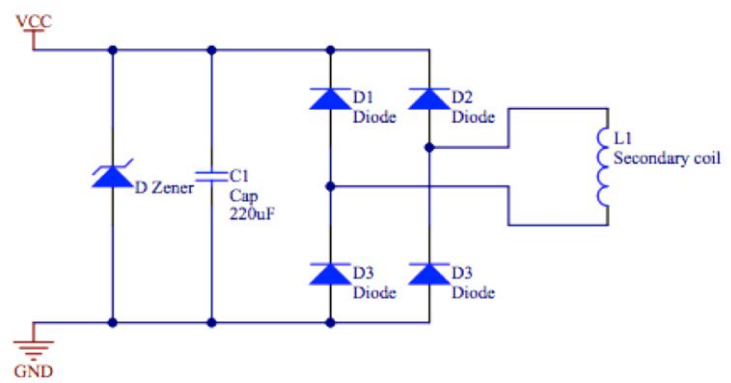

Fig 6: AC to DC Conversion Circuit

The schematic for AC to DC conversion circuit is shown above. The four diodes that are connected to the inner coil form a full wave rectifier that is able to convert $\mathrm{AC}$ input into voltage with only one polarity. This single polarity voltage gets stored in a $220 \mathrm{uf}$ capacitor, and a zener diode works as a voltage regulator to stabilize the output voltage of the capacitor to 5 voltages. The 5 volt DC voltage is fed into the Vec port of microcontroller to provide power.

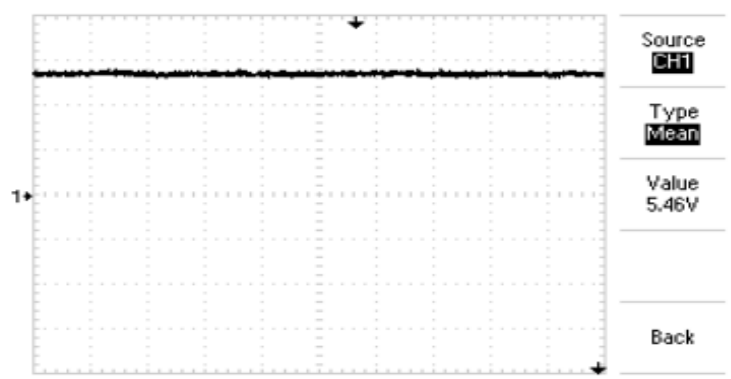

Fig7: Voltage of Secondary Coil after AC to DC Conversion

\subsubsection{LED Array}

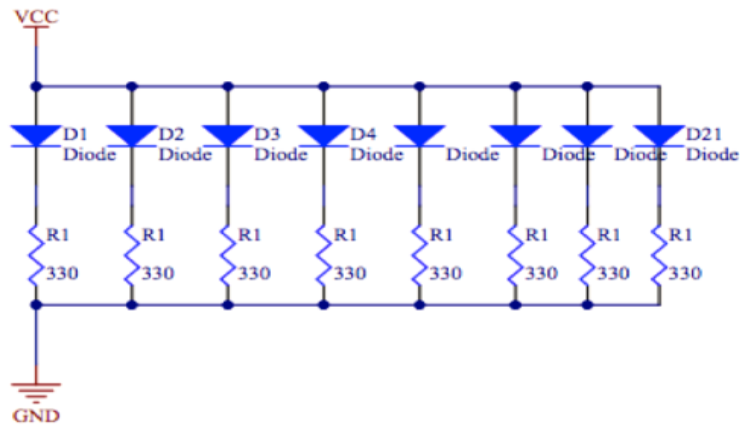

Fig8: LED Array 
There is a row of LEDs on one side of the rotor. Each LED's positive lead is connected to a pin on microcontroller and negative lead is connected to ground via a $330 \mathrm{ohm}$ resistor. The $330 \mathrm{ohm}$ resistor is there for protecting the LED. There are 15 LEDs in total, out of which 14 blue colored and one are is red colored. The red colored LED is the outmost LED and is responsible for producing the outer frame of the display. It is constantly on once the power-on button is pressed. The LEDs are mapped to all pins for PORTA and PORTB, and some pins of PORTC. Two ports on this microcontroller have 6 pins and one port has 8 pins [2].

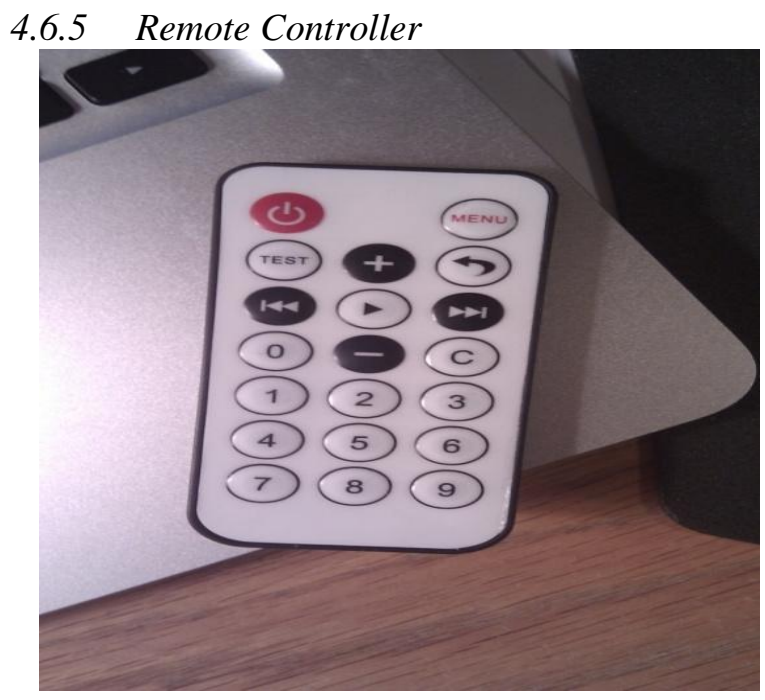

Fig9: Remote Control

Another piece of hardware is the remote controller. The remote control used is a standard 38k IR remote controller. This is used because its behavior is well understood and decoding code examples are widely availed online.

\subsubsection{IR Receiver Circuit}

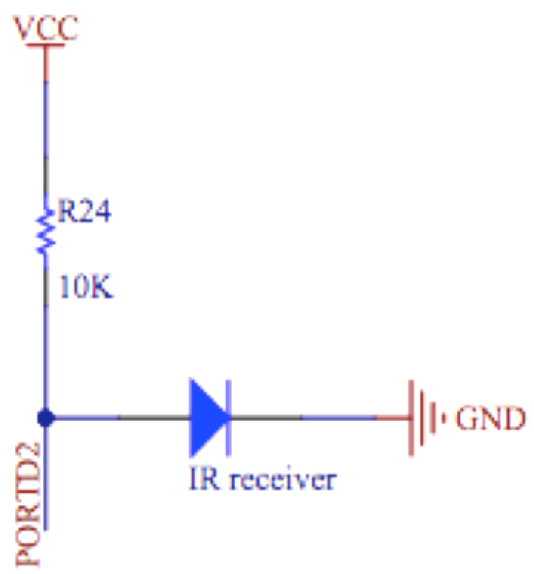

Fig10: IR Receiver Circuit

The IR receiver corresponding to the IR transmitter in motor circuit is connected to Vcc through a $10 \mathrm{k}$ resistance. $10 \mathrm{k}$ resistor lowers the current in that branch. The positive lead of the receiver is connected to Vcc via a resistance and the external interrupt port PORTD while the negative lead is connected to ground. Under normal operation, the receiver is open. PORTD is always high. When it receives from the transmitter, it closes and pulls PORTD low. The external interrupt 0 is trigger on falling edge. It is used to calculate cycle time.

\section{FLOW CHART}

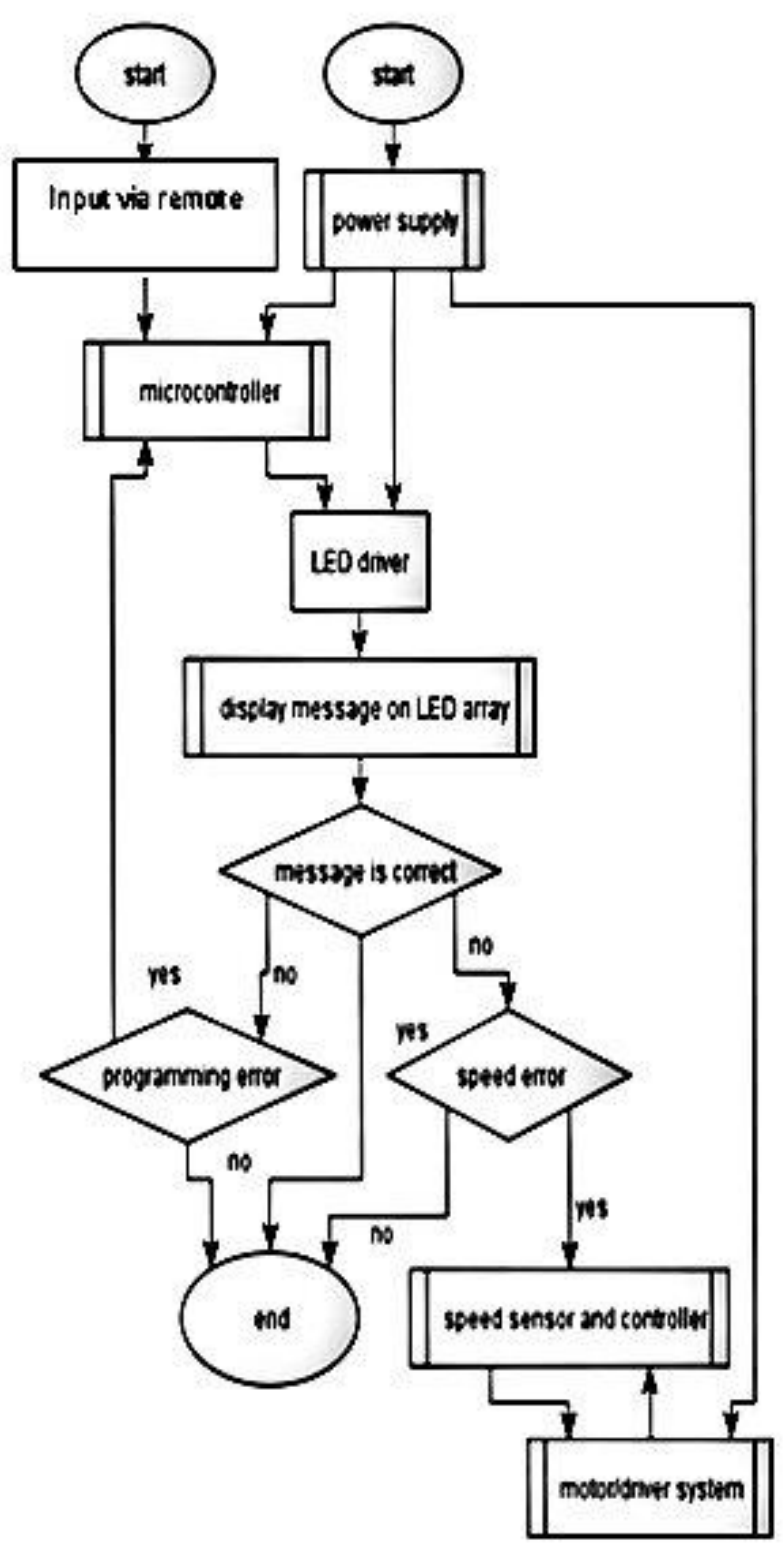

Fig11: Flow of system

\section{CONCLUSION}

This paper introduced a wireless remote controlled persistenceof-vision (POV) display that is just simple enough and robust and the wireless mechanism makes it more durable and detachable. It also achieves the desired POV effect that looks so cool and can be used for effective message displays. This project really demonstrated competence combining a difficult integration of the mechanical and electrical systems to build a persistence of vision display. This project also has so much room to explore further exciting developments and additions to the many devices with which it could interface. It also has been made sure that this project would not cause any ethical concerns. However, the simplistic design has its trade-offs; especially in terms of reliability. This project is basically showing 2 dimensional figures from 1 dimensional array of led's and the project can be expanded further to make it more 
interactive and user-friendly by implementing certain changes such as:

1. Using multiple microcontrollers to view POV of large sizes.

2. LED controllers can be used to change intensity of led's and can be used for showing high quality images.

3. Rotating a 2-D array of led setup, cool 3-D views can be made which can be viewed from all the directions.

4. Upgrading the display module and using speed controlled motor would allow us to improve the display with minimal changes.

5. Expand the radius of the device to display a longer message. Also can consider expanding to more LED's.

6. Addition of various text effects

7. Furthermore, 3D effects can also be incorporated in the display.

8. This display can be programmed as an Analog clock.

\section{FUTURE SCOPE}

Advertisement: In this modern era there is a growing need of advertising the products \& services. People get influenced by good advertisement. Persistence of vision proved to be a very effective method for providing a visual image to attract the consumers. Advertisement can be made much more attractive by two dimensions or three dimension visual image.

Entertainment: Persistence of vision plays a very vital role in entertainment point of view. It can be used in shopping malls for displaying messages. It can also be used in film industry. Motion pictures are based on the principle of persistence of vision. The display is like any complex toy.We can make it for fun and diversion by using this application in games and toys.

Animation: The Persistence of vision is the basic principle of animation. This technique is also used in cartoon animation. Cartoon movies are also the result of persistence of vision. Education: If Persistence of vision is used in education field it will provide a better way for interactive education. Many concept need to be explained in three dimension view that can be easily demonstrated by the help of this persistence of vision.

\section{DISPLAY GENERATED PATTERNS}

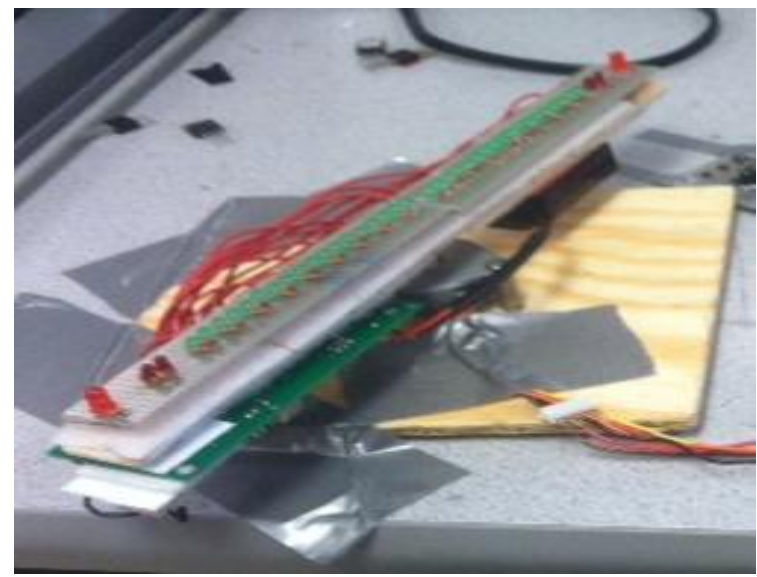

Fig12: Actual hardware module

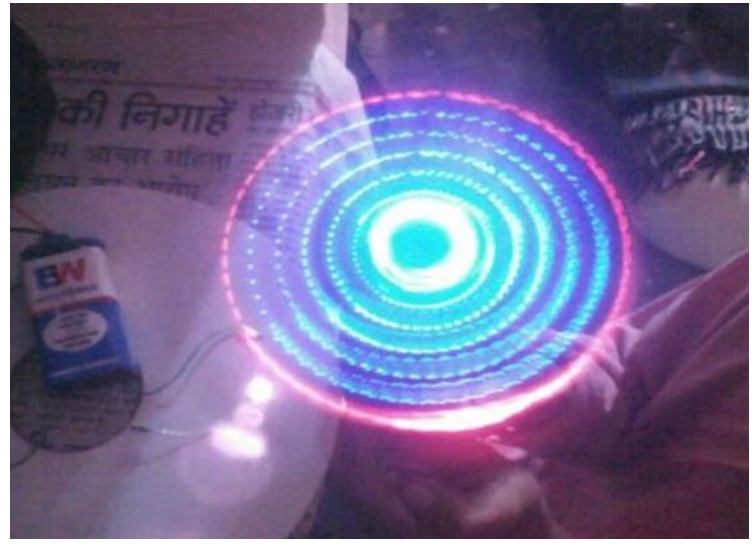

Fig13: Displaying a complete circle

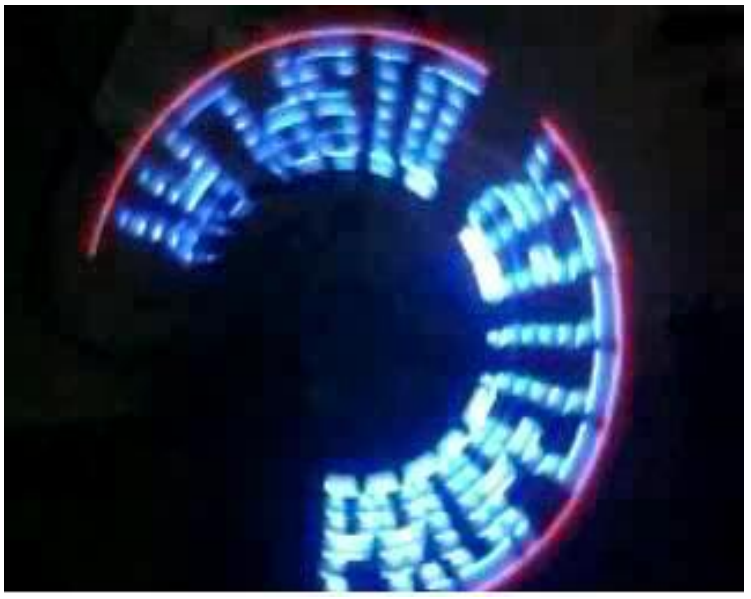

Fig14: Displaying a String

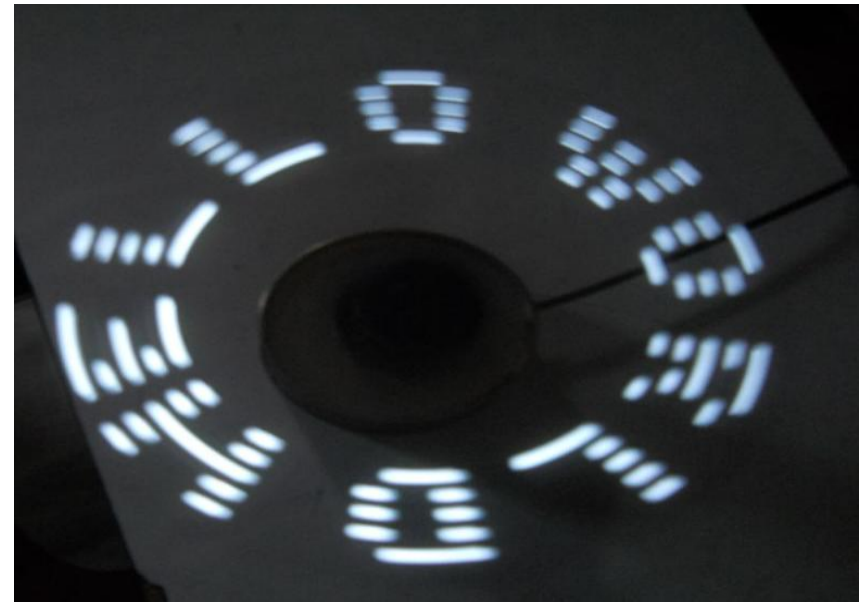

Fig15: Displaying a Message

\section{ACKNOWLEDGEMENTS}

We owe a great many thanks to a great many people who helped and supported us during the making of this project.

Deepest thanks to our project guide Prof. Shivani Bhattacharjee for guiding and correcting various documents of this project with attention and care. She has taken pain to go through the project and make necessary correction as and when needed.

We express our thanks to Dr. Hari Vasudevan. Principal of Dwarkadas J. Sanghvi College of Engineering, Mumbai, for extending his support. My deep sense of gratitude to our 
HOD Dr. Amit Deshmukh for support and guidance. We would also thank our Institution and our staff members without whom this project would have been a distant reality. We also extend our heartfelt thanks to our family and well wishers.

\section{REFERENCES}

[1] Muhammad AliMazidi. Janice GillispieMazidi and Rolin D. McKinlay, the 8051 Microcontroller and Embedded System Using Assembly and C, 2nded, Pearson, 2007.

[2] Remote Controlled POV Display from http://people.ece.cornell.edu/land/courses/ece4760/Final Projects/f2013/js2839_yc969/js2839_yc969/js2839_yc9 69/index.html

[3] Global Journal of researches in engineering Electrical and electronics engineering Volume 12 Issue 4 Version 1.0 March 2012 "The Design and Construction of a low cost Propeller Led Display" By Sheikh Rafik Manihar, Mr. Komal Prasad Dewangan \& Mr. Ajay Kumar Dansena mentor ARPIT NEAM(IIT Kanpur).
[4] Coltheart M. "The persistence of vision." Philos Trans R SocLond B Biol Sci. 1980 Jul 8; 290(1038):57-69. PMID 6106242.

[5] Technical reference detailing the LED display array, RF interface and scanning circuit was included as part of the 1978 29th ISEF exhibition in Anaheim, CA.

[6] Mitchell ${ }^{\text {ees }}$ modular LED x-y (horizontally and vertically digitally scanned array system) was cited in the 29th International Science and Engineering Exposition "book of abstracts", page 97, published by the "Science Service", Washington D.C. May1978.

[7] Cook, David A. (2004) A History of Narrative Film. New York, W. W. Norton \& Company.

[8] International Journal of Advanced Research in Electrical, Electronics and Instrumentation Engineering (An ISO 3297: 2007 Certified Organization) Vol. 3, Issue 2, February 2014.

[9] Limor's MiniPOV project [www.ladyada.net] 